\section{La Révolution française}

Cahiers de l'Institut d'histoire de la Révolution française

$6 \mid 2014$

La Révolution ou l'invention de la femme et de l'homme nouveaux

\title{
Réformer le peuple français : la création du citoyen révolutionnaire et le rôle des institutions dans les œuvres de Saint-Just
}

\section{Marylin Maeso}

\section{OpenEdition}

\section{Journals}

Édition électronique

URL : http://journals.openedition.org/lrf/1093

DOI : $10.4000 /$ Irf. 1093

ISSN : 2105-2557

Éditeur

IHMC - Institut d'histoire moderne et contemporaine (UMR 8066)

Référence électronique

Marylin Maeso, «Réformer le peuple français : la création du citoyen révolutionnaire et le rôle des institutions dans les œuvres de Saint-Just », La Révolution française [En ligne], 6 | 2014, mis en ligne le 28 septembre 2014, consulté le 14 février 2020. URL : http://journals.openedition.org//rf/1093 ; DOI : 10.4000/Irf.1093

Ce document a été généré automatiquement le 14 février 2020.

(C) La Révolution française 


\title{
Réformer le peuple français : la création du citoyen révolutionnaire et le rôle des institutions dans les œuvres de Saint-Just
}

\author{
Marylin Maeso
}

Les écrits politiques de Saint-Just témoignent d'une conscience aiguë des tensions inhérentes à toute révolution, rejeton nécessairement prématuré de l'histoire. Nécessairement, car une rupture radicale avec un ordre social et politique séculaire ne peut, sous peine d'être étouffée dans l'œuf par les tenants du régime déchu, procéder par étapes et méthodiquement: elle ne peut triompher qu'en se faisant «coup de tonnerre sur tous les méchants ${ }^{1} »$, pour reprendre le mot de celui que l'on a surnommé l'Archange de la Terreur. Prématuré, parce que le phénomène révolutionnaire surgit au sein d'une société façonnée par un système politique, des mœurs, des traditions et des symboles qu'il s'agit de balayer pour leur substituer un nouveau régime qui apporte avec lui de nouvelles pratiques et une idéologie rigoureusement élaborée en opposition à celle du régime précédent. Mais dès lors que la République à bâtir se veut l'antithèse parfaite du régime monarchique, comme la justice et la vertu s'opposent à la corruption et à l'iniquité, la révolution qui doit en hâter l'avènement ne peut qu'être totale et fulgurante. Il faut, pour ainsi dire, métamorphoser les Français, faire d'un troupeau d'esclaves abêtis par des siècles de domination un peuple de citoyens libres. Il faut façonner les esprits à l'image de la société heureuse et pure que les hommes de 1789 appellent de leurs vœux. Telle est, une fois la monarchie abolie, l'ambition démesurée et éminemment problématique des révolutionnaires: bâtir un nouveau système sur les ruines vermoulues et fragiles du précédent ; forcer les anciens sujets du roi à être libres, les ramener malgré eux à la raison et à la morale, alors même qu'ils adorent leurs anciennes chaînes et voient pour la plupart dans la Révolution au mieux un bouleversement effrayant des habitudes et d'un habitus confortables, au pire, le mal absolu qu'il faut éradiquer à tout prix : «Il faut voir avec quelle pénétrante sagesse

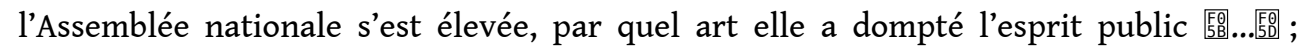


comment elle a ingénieusement enchaîné le peuple de sa liberté, l'a étroitement lié à la Constitution en érigeant ses droits en maximes, et en séduisant ses passions ${ }^{2}$. " C'est dans ces moments où la guerre des dieux fait rage que la violence, éternelle accoucheuse de l'Histoire, semble s'imposer comme seule à même de trancher les nœuds gordiens.

2 A l'instar des autres révolutionnaires, Saint-Just est habité par le souci permanent de donner à la République naissante des hommes dignes d'elle, par le constat triste mais inévitable de l'incapacité de l'écrasante majorité de ses contemporains à accueillir, en l'état, un changement d'atmosphère aussi radical. Tel le prisonnier fraichement libéré de la caverne platonicienne et aveuglé par la vérité solaire qui l'attend au bout de l'ascension, le peuple français tremble devant l'univers inconnu qui s'ouvre à lui, il a besoin d'un guide pour l'aider à franchir le pas, d'un éducateur pour l'éclairer et lui faire comprendre les raisons qui doivent l'amener à le franchir. Cette thématique pédagogique, centrale dans les écrits de Saint-Just, est trop souvent oblitérée par la réputation qui précède l'auteur, celle d'un disciple admiratif de Robespierre tout aussi virulent que lui lorsqu'il s'agit de prôner le rôle bénéfique de la Terreur. Or, sur ce point précis, il importe de remarquer que la position de Saint-Just est plus complexe que celle de son aîné. Là où Robespierre justifie la Terreur comme méthode légitime qui s'impose pour fonder la République ${ }^{3}$, Saint-Just, quant à lui, ne va pas jusqu'à identifier vertu et Terreur: il en fait bien plutôt les deux branches d'une alternative. "Un gouvernement républicain ", selon lui, " a la vertu pour principe ; sinon la terreur ${ }^{4}$." Un peu plus loin, il précise qu' " il faut dans toute révolution un dictateur pour sauver l'Etat par la force ou des censeurs pour le sauver par la vertu ${ }^{5}$.» Ces propos tendent à montrer que Saint-Just continue à nourrir, au moment même où la Terreur est devenue la politique officielle du gouvernement $t^{6}$, l'espoir d'une transformation intérieure des Français, d'un renouvellement complet des mœurs à même d'ancrer l'amour de la République dans le cœur de chacun. La solution qu'il préconise est donc essentiellement pédagogique : il s'agit de réformer le peuple français et de former le citoyen républicain.

3 Afin de restituer fidèlement le projet de Saint-Just, il convient de comprendre le terme de « réforme » en son sens étymologique : il désigne, sous la plume de notre auteur, l'action de retrouver, de restaurer sous la couche épaisse de corruption monarchique, la pureté originelle de la nature humaine, de nettoyer, pour reprendre, mutatis mutandis, une célèbre image rousseauiste, la statue de Glaucus. En effet, contrairement aux représentations que la notion d' "homme nouveau " éveille spontanément, le citoyen modèle que Saint-Just appelle de ses vœux, loin d'être le fruit d'un progrès de l'histoire menant de la monarchie à la République, doit être directement issu de la résurrection de l'homme social sans tâche des temps apolitiques, comme si la Révolution, mettant un terme à la succession de malheurs qu'est l'histoire, avait pour effet salutaire de ramener le peuple français aux origines de la société. Renouant avec son étymologie latine, la Révolution formerait ainsi un retour à un état de félicité sociale enseveli sous une oppression politique séculaire.

Ces considérations montrent nettement qu'aux yeux de Saint-Just, la véritable Révolution doit se faire dans le cœur des hommes. La théorisation de cette réforme sociale et morale s'effectue principalement au sein de deux œuvres qui nous semblent particulièrement pertinentes pour comprendre la position de cet auteur concernant la formation de l'homme nouveau : dans la première, intitulée De la nature, de l'état civil, de 
la cité ou les principes de l'indépendance, du gouvernement, Saint-Just établit le diagnostic de la maladie politique qui ronge le peuple français, et insiste sur la nécessaire purification qui doit présider au bon rétablissement du corps social dans son état de santé originel. Dans un deuxième temps, il entreprend la rédaction d'Institutions Républicaines (manuscrit inachevé qui constitue le dernier texte écrit par lui), texte dans lequel il expose un certain nombre de mesures positives visant à fédérer le tissu social en tentant de recréer autant que faire se peut ${ }^{7}$ l'harmonie sociale qui selon lui animait les communautés humaines primitives ${ }^{8}$ : au diagnostic alarmant répond l'énoncé de la cure. Nous allons donc tâcher de suivre la logique du raisonnement qui fédère ces deux textes, en commençant par expliciter les raisons qui appellent de manière urgente, pour Saint-Just, une renaturation des mœurs et des esprits, avant de réfléchir aux mesures (ou aux miracles républicains) qui peuvent concrétiser un tel projet. Ces réflexions nous amèneront, pour finir, à relever un certain nombre de tensions et d'apories qui traversent les textes analysés, afin de mettre en lumière l'exceptionnalité et la démesure qui caractérisent l'ambition anthropogonique ${ }^{9}$ de Saint-Just.

5 Commençons par une précision : le choix que nous faisons de nous concentrer sur les deux derniers écrits théoriques de Saint-Just, s'il se justifie par la teneur de ces textes (ils comportent la thématisation la plus riche et la plus précise de l'idée de la corruption de la société française et de la nécessaire réforme sociale et morale qui en découle), ne doit pas suggérer que ce souci de formation de l'homme nouveau n'occupe l'auteur que dans une période secondaire de sa carrière intellectuelle et politique. Dès son essai de juin 1791 intitulé L'esprit de la Révolution et de la constitution de la France, il esquisse les prémisses de son anthropologie et de sa théorie morale. D'emblée, le ton est donc donné : il conçoit l'évolution des hommes et de leurs mœurs comme un long processus de décadence qui part d'un état naturel postulé, innocent et heureux, et qui sombre sous l'effet d'une dénaturation progressive de cette pureté humaine originelle : "Les mœurs sont les rapports que la nature avait mis entre les hommes; ils comprennent la piété filiale, l'amour et l'amitié. Les mœurs dans la société sont encore ces mêmes rapports mais dénaturés. La piété filiale est la crainte; l'amour, la galanterie ; l'amitié, la familiarité. Une constitution libre est bonne à mesure qu'elle rapproche les mœurs de leur origine, que les parents sont chéris, les inclinations pures et les liaisons sincères ${ }^{10}$. » Le manuscrit De la nature reprend ce constat de façon plus systématique. En partant de l'état alarmant de la société française actuelle, Saint-Just reconstitue rétrospectivement le portrait de la société primitive qui apparaît comme le modèle, l'horizon vers lequel doivent tendre les éducateurs des citoyens républicains de demain. Il relève de façon très précise ce qui a provoqué selon lui la chute hors de la communauté primitive : il s'agit d'une substitution fatale, au sein de la société, de principes politiques (la domination, la violence : l'idée même d'un contrat social qui obligerait artificiellement les citoyens les uns envers les autres constitue pour SaintJust une forme de coercition inacceptable et incompatible avec les principes dictés par la nature ${ }^{11}$ ) aux principes sociaux (la liberté, l'harmonie entre les individus) : "pour avoir confondu le droit social et le droit politique, on a fait des agrégations et non point des sociétés [...] la république a fait un contrat politique ou de force entre chacun et tous et ce contrat politique forme un pacte social. Mais quelle violence et quelle faiblesse, tandis que la nature est là, qu'on oublie et qu'on outrage! Ces sociétés ressemblent à des traités de pirates qui n'ont de garants que le sabre. Les flibustiers avaient aussi un pacte social sur leurs navires ${ }^{12}$.» La vigueur de ces affirmations rend 
palpable l'ampleur de la tâche de réforme sociale qui attend les révolutionnaires. Les Français ont été littéralement dénaturés par des siècles de régime monarchique, ils ont pris le pli de la servitude, se sont habitués à l'oppression comme si celle-ci était naturelle, et ont ainsi oublié jusqu'à l'essence même de leur nature sociale. Pour le dire avec les mots de Saint-Just: «L'homme s'ignore soi-même et tout fier de la fausse nature qu'il s'est faite, comment retrouvera-t-il la véritable ${ }^{13}$ ?»

C'est donc un travail de purification des mœurs et des cœurs qui doit être entrepris afin de faire resurgir cette essence sociale originelle trop longtemps étouffée et trahie par les ennemis de la Révolution. Tout semble indiquer qu'aux yeux de notre auteur, c'est à partir de ce moule ancestral qu'il faudra modeler l'homme nouveau, seul à même de perpétuer l'héritage révolutionnaire. Mais c'est justement là que gît la difficulté principale de son projet de société, et il convient de donner toute sa force problématique à la question que nous venons d'évoquer : "L'homme s'ignore soi-même et tout fier de la fausse nature qu'il s'est faite, comment retrouvera-t-il la véritable ${ }^{14}$ ?» De fait, l'homme moderne étant un être non seulement dénaturé, mais incapable de recouvrer sa pureté perdue, tant le processus de corruption a bouleversé les sociétés et les individus, comment pourrait-on espérer faire renaître cette socialité primitive dans le cœur des hommes? Saint-Just, visiblement conscient de cet obstacle, ne songe pas à restaurer purement et simplement la communauté originelle, et l'on ne peut manquer de relever qu'il reconnaît en toute lucidité que des siècles d'histoire ainsi que les évolutions qu'ils ont imprimées au corps social ne peuvent être biffés : «Hélas, il ne faut plus songer à faire goûter aux hommes l'attrait de l'innocence, on ne ferait plus que des êtres faibles et dépravés; la vertu même n'est plus innocente ${ }^{15}$.» Et il en tire les conséquences dans un discours postérieur : «Permettez-moi de développer mes idées ; elles amènent ce par où je dois conclure : je saurai les plier à l'ordre des choses, et je ne refuserai pas à la loi la force dont elle a besoin en prenant l'homme tel qu'il est ; mais je conçois un gouvernement vigoureux et légitime ; il ne faut point songer à la politique naturelle, et ce n'est point là mon idée ${ }^{16}$.»

7 A ce stade de la réflexion de Saint-Just, on se trouve confronté à un paradoxe qui menace de déboucher sur une aporie : comment peut-on vouloir régénérer les mœurs des citoyens, former des hommes nouveaux en accord avec leur nature véritable (c'està-dire vertueux et solidaires) et en même temps admettre, avec les accents du désespoir, que cette vertu est devenue inaccessible? Et pourquoi prétendre abolir le règne de la politique qui est celui de la force et de l'oppression, si c'est pour recourir à une violence peut-être plus destructrice encore (celle de la Terreur) en vue de réaliser les ambitions révolutionnaires? Peut-on véritablement transformer la nature humaine, lui redonner la spontanéité et l'harmonie qui caractérisent l'état social premier, en usant de la contrainte policière ${ }^{17}$ ?

Cette tension, Saint-Just semble l'avoir nettement perçue : si la politique et la violence sont synonymes de corruption, si elles sont les fléaux responsables de la destruction de l'état de nature social et pacifique, il devient difficile de se résoudre sans hésitation à recourir à des méthodes terroristes. Vouloir soigner le mal par le mal est une entreprise pour le moins périlleuse, et c'est la raison pour laquelle Saint-Just, dans ses Institutions républicaines, entend élaborer une autre voie pour instaurer définitivement le règne de la vertu en faisant l'économie de la Terreur. Comment changer l'homme, le ramener au plus près de la nature (puisque la communauté originelle est quant à elle à jamais perdue), sans lui faire violence? En forgeant des institutions qui soient 
l'émanation directe d'un ordre objectif susceptible d'assurer leur légitimité, à savoir la nature. Dans une perspective éminemment rousseauiste, notre auteur remet ainsi le salut du peuple français entre les mains du Législateur qui a pour noble mission de pétrir les mœurs humaines pour leur donner, grâce à sa sagesse, le pli de la vertu : «quand j'ai pénétré l'esprit du législateur, j'ai vu sortir l'ordre du chaos, les éléments se séparer et créer la vie ${ }^{18}$ »; «Le législateur commande à l'avenir ; il ne lui sert de rien d'être faible: c'est à lui de vouloir le bien et de le perpétuer; à lui de rendre les hommes ce qu'il veut qu'ils soient : selon que les lois animent le corps social inerte par lui-même, il en résulte des vertus ou les crimes, les bonnes mœurs ou la férocité. La vertu de Lacédémone était dans le cœur de Lycurgue et l'inconstance des Crétois dans le cœur de Minos $^{19}$.» Loin de vouloir imposer aux Français, à l'instar d'un dieu monarque ou du roi de droit divin ${ }^{20}$ que les révolutionnaires viennent de détrôner, une forme légale qui leur soit extérieure et étrangère, le Législateur doit rétablir l'ordre objectif des choses en prenant pour base les hommes tels qu'ils sont et les ramener avec douceur vers la pureté de leur nature : "lorsqu'une révolution change tout à coup un peuple, et qu'en le prenant tel qu'il est on essaye de le réformer, il se faut ployer à ses faiblesses, et le soumettre avec discernement au génie de l'institution; il ne faut point faire qu'il convienne aux lois, il vaut mieux faire en sorte que les lois lui conviennent. Notre constitution doit être propre au peuple français. Les mauvaises lois l'ont soumis longtemps au gouvernement d'un seul: c'est un végétal transporté dans un autre hémisphère qu'il faut que l'art aide à produire des fruits mûrs sous un climat nouveau ${ }^{21}$.» Cette solution, en plus de supprimer l'arbitraire de la domination, a le mérite de dédramatiser l'aporie qui s'était dessinée auparavant : il n'est pas question ici de recréer la communauté sociale primitive, mais seulement de prendre les caractéristiques inhérentes à la nature vraie des hommes comme modèle pour les institutions à forger. Et c'est précisément à partir du moment où l'on admet que l'état de nature est à jamais perdu et que «dans cet état où nous vivons, la vie civile est la plus naturelle [...], celle qu'il faut cultiver avec le plus de soin", que l'on pourra se rapprocher au plus près de cet éden disparu. La métaphore botanique convoquée par Saint-Just est à cet égard éclairante: le peuple français, propulsé en l'espace de quelques années dans un univers politique et idéologique qui lui est radicalement étranger, a besoin d'un temps d'acclimatation, il lui faut l'aide des chefs de file de la Révolution pour apprendre progressivement comment redevenir vertueux et devenir républicain. En institutionnalisant la vertu, c'est-à-dire en inculquant par «l'art » ce que la nature avait produit mais que la monarchie a balayé, le gouvernement doit instiller dans le cœur des citoyens les valeurs sociales qui font l'excellence de la nature humaine. Quelles sont ces valeurs? Force est de constater que l'esprit qui préside à l'élaboration de ces institutions est placé sous le signe de l'ascétisme spartiate et des valeurs phares de la cité grecque antique (du moins celle que Platon aurait souhaité bâtir). C'est en effet la solidarité des citoyens entre eux, et plus précisément l' " amitié ", qui est élevée au rang de règle d'or de la nouvelle société française. SaintJust insiste continuellement dans ses divers écrits sur l'importance de l' « union » et de l' « harmonie » comme puissances constitutives de la société, en précisant notamment que le gouvernement ne doit pas être une instance autoritaire mais un «ressort $\mathrm{d}^{\prime}$ harmonie ${ }^{22}$ » pour le peuple. Ainsi, l'auteur cherche paradoxalement à institutionnaliser ce qui semble plutôt relever du sentiment spontané : «Celui qui dit qu'il ne croit pas à l'amitié est banni. Tout homme âgé de 21 ans est tenu de déclarer dans le temple quels sont ses amis et cette déclaration doit être renouvelée tous les ans 
pendant le mois de ventôse [...]. Si un homme commet un crime, ses amis sont bannis [...] Si un homme n'a point d'amis, il est banni ${ }^{23}$. » C'est que l'amitié, telle que la conçoit Saint-Just, est moins un sentiment subjectif qu'un ferment de lien social, en ce qu'elle permet de maintenir la communauté soudée, et c'est pourquoi tout citoyen se doit de la cultiver et de la protéger, sous peine d'être frappé d'indignité par la société ${ }^{24}$. Le jeune citoyen doit donc être élevé dans un esprit communautaire (" c'est vraiment alors que la République est une et indivisible et que le souverain se compose de tous les cœurs portés à la vertu $\left.{ }^{25} »\right)$ qui fait primer la collectivité sur l'individu, contre l'égoïsme, l'individualisme et l'esprit de rivalité maladive qui ont, selon notre auteur, rongé la société monarchique : «C'est donc à vous, Législateurs, de poser sans cesse la question du bien public, d'en rapprocher tout, d'y soumettre tout ce qui se dit et se fait; par là vous conserverez votre influence, par là vous jugerez les passions qui nous sont contraires, par là vous substituerez dans l'Etat, le génie commun de la patrie à la jalousie et aux cris des factions ${ }^{26}$.» L'enjeu est de rassembler les conditions de possibilité d'une république unanime au sens le plus fort du terme : les citoyens doivent se comporter comme s'ils étaient animés par une seule âme. Chaque membre du corps social doit répondre de ses choix et de ses actions devant l'ensemble de la communauté. Ainsi, «ceux qui se brouillent sont tenus d'en expliquer les motifs devant le peuple, dans les temples, sur l'appel d'un citoyen ou d'un plus vieux, s'ils le refusent ils sont bannis $^{27}$ », car il importe au plus haut point de s'assurer que ce qui apparaît au premier abord comme une simple dispute entre individus ne puisse dégénérer en lutte fratricide et mettre par là en danger l'avenir de la République. Sur la base de ce consensus social extrême et contrôlé, c'est tout un réseau de règles structurantes qui s'instaure et fédère la vie des citoyens dans toutes ses dimensions: ainsi le courage et un patriotisme inflexible seront eux aussi des vertus indispensables au citoyen républicain («Un militaire ne peut jamais rentrer dans le lieu où il est né, s'il a quitté son rang dans les combats, s'il a perdu son arme, s'il a déserté, s'il a violé la discipline, s'il a murmuré des fatigues $^{28} »$ ), puisque ces qualités sont nécessaires à la défense de la cité contre les assauts de l'étranger. Mais c'est surtout sur l'éducation des enfants que l'accent est mis, car c'est dès le plus jeune âge que l'homme nouveau doit être façonné dans son cœur et dans ses mœurs afin qu'il intériorise l'esprit républicain. Puisque les enfants nés sous la République n'ont pas connu la corruption monarchique, ils seront d'autant plus réceptifs à la vertu qu'en eux la nature est demeurée pure. L'éducation doit, pour ce faire, être assurée par l'Etat (il est indiqué que les enfants « ne retournent jamais chez leurs parents avant l'âge de 21 ans $^{29} »$ ), et les mesures qui la réglementent ne sont pas sans rappeler de célèbres passages de la République de Platon: nourriture simple et rustique, vêtements de toile; éducation militaire (et agricole : ici l'influence du milieu où Saint-Just a grandi, c'est-à-dire les petites communautés rurales très soudées de l'Allier, se fait sentir), autant d'aspects typiquement spartiates que notre auteur emprunte clairement à ses lectures classiques. Cette éducation est d'emblée centrée sur l'inculcation de savoir-faire, et doit se dérouler sur une durée suffisamment longue pour s'assurer que l'habitus républicain soit complètement enraciné en eux et constitue pour eux comme une seconde nature: "Les laboureurs, les manufacturiers, les négociants sont instituteurs [...]. Les jeunes hommes de 16 ans sont tenus d'être chez les instituteurs jusqu'à 21 ans, à peine d'être privés du droit de citoyen pendant leur $v^{3 i}{ }^{30}$.» Ce sont les sages (c'est-à-dire, parmi les hommes pratiquant les métiers énumérés ci-dessus, ceux qui sont âgés d'au moins soixante ans, qui ont reçu l'écharpe de la vieillesse et qui ont été élus par le peuple) qui doivent s'en charger, ce qui 
souligne le sérieux avec lequel Saint-Just entend organiser la formation des jeunes citoyens français, tant il est convaincu que l'enfant, plus proche de la nature que ne l'est l'adulte, n'a pas encore été happé par la corruption qui régnait dans la société d'Ancien régime : il est la promesse du renouveau social que Saint-Just appelle de ses vœux. Ne déclare-t-il pas, au tout début de L'esprit de la révolution : " parce que j'étais jeune, il m'a semblé que j'en étais plus près de la nature ${ }^{31}$ ?"

On réalise ici tout l'espoir que Saint-Just fonde sur l'instauration de ces institutions républicaines : elles constituent à ses yeux la seule alternative envisageable à la Terreur pour réformer un Etat corrompu, idée qu'il expose à de nombreuses reprises ( «Il faut dans toute révolution un dictateur pour sauver l'Etat par la force ou des censeurs pour le sauver par la vertu ${ }^{32}$ »; « un gouvernement républicain a la vertu pour principe, sinon la terreur ${ }^{33}$ "; "La révolution est glacée; tous les principes sont affaiblis; il ne reste plus que des bonnets rouges portés par l'intrigue. L'exercice de la terreur a blasé le crime comme les liqueurs fortes blasent le palais $\left.{ }^{34} »\right)$. Les institutions sont donc bien l'instrument de la renaturation des citoyens français : "S'il y avait des mœurs, tout irait bien ; il faut des institutions pour les épurer. Il faut tendre là : voilà tout ce qu'il faut faire ; tout le reste s'ensuivra. La terreur peut nous débarrasser de la monarchie et de l'aristocratie ; mais qui nous délivrera de la corruption? Des institutions $\mathbf{s}^{35}$.» De fait, ces dernières ont pour vertu de pouvoir pénétrer les corps (par le biais de l'éducation physique et militaire, ainsi que par la simplicité de l'alimentation et de l'habillement) tout autant que les esprits (par la mise en place d'un culte religieux civique, et par la diffusion d'une idéologie mettant le bonheur commun et la perpétuation de la société au-dessus de toute autre fin) sans les contraindre, et c'est bien là leur supériorité par rapports aux lois: " une loi contraire aux institutions est tyrannique [...]. Les longues lois sont des calamités publiques [...]. Il faut peu de lois. Là où il y en a tant, le peuple est esclave [...]. Le nom de loi peut sanctionner le despotisme; le despotisme est l'exercice sur le peuple d'une volonté extérieure à la sienne ${ }^{36}$.» En ce qu'elles misent sur l'intériorisation d'un état d'esprit et d'un mode de vie républicains plutôt que sur l'imposition extérieure de lois qui imposent l'obéissance sans transformer les cœurs, les institutions tracent pour Saint-Just la voie la plus féconde vers l'instauration d'une communauté aussi fidèle que possible à la nature humaine lavée des stigmates du régime monarchique.

Cette analyse ne serait pas complète sans que soient au moins esquissés les tensions et les obstacles que le projet pédagogique de Saint-Just ne manque pas de soulever, du fait de son caractère radical et intempestif. Il nous semble que, pour l'essentiel, ces difficultés peuvent être subsumées sous un problème fondamental qui sera notamment thématisé par Hegel au siècle suivant, à savoir celui du changement profond d' "esprit » qui sépare à jamais les cités antiques des sociétés modernes. Ce dernier note en effet, dans sa Philosophie de l'Esprit. 1805, que la totalité éthique que forme la cité grecque, si elle est admirable par la parfaite cohésion qu'elle réalise, ne fait néanmoins plus sens dans le cadre des Etats que nous connaissons, dans la mesure où le " principe supérieur des temps modernes que les anciens, que Platon ne connaissaient pas $^{37}$ ", à savoir le respect de l'individu, de ses aspirations personnelles et de sa vie privée, implique que la singularité des citoyens d'aujourd'hui ne puisse être purement et simplement sacrifiée au profit d'une "unité immédiate de l'universel et du singulier ${ }^{38}$ " telle qu'avait pu la réaliser la vie civique grecque. Or, force est de constater, à la lecture des Institutions républicaines, que Saint-Just tend à nier l'autonomie de la sphère privée et sa valeur propre, en cherchant à l'institutionnaliser 
et à la réguler tout autant que la sphère publique, à tel point que la distinction entre ces deux domaines semble effacée. Car si Saint-Just soutient qu'il faut renoncer au rêve de ressusciter l'état de nature, il semble bien que son projet repose sur la postulat qu'il est néanmoins possible de faire revivre, dans la France révolutionnaire, le modèle de la république romaine, autrement dit sur l'idée qu'on peut effectivement réaliser une révolution au sens étymologique du terme: un retour aux sources véritables de la vie sociale que l'auteur situe dans l'Antiquité, pari pour le moins problématique, comme en témoignera la tournure prise par Révolution française au fil de son évolution.

11 Au terme de cette étude succincte des travaux de Saint-Just, il apparaît clairement que la formation de l'homme nouveau est au cœur de ses préoccupations dès 1791. Le fait qu'il consacre son dernier écrit à cette même question confirme l'importance de l'enjeu : former les citoyens de la République, c'est se donner les moyens d'enraciner ce nouveau régime et d'assurer sa pérennité. Toute la difficulté du projet repose sur ce qui en constitue le point nodal, à savoir la volonté d'unifier les Français autour d'idées et de valeurs communes, à une époque où la France demeure encore une mosaïque de terroirs à la diversité culturelle très marquée. L'intérêt des écrits de Saint-Just n'en est que plus évident, puisque cette nécessité de républicaniser les Français et de créer progressivement, en douceur, un sentiment d'appartenance à une même nation, sera, mutatis mutandis, le mot d'ordre des Républicains au XIX ${ }^{e}$ siècle et plus particulièrement de l'école républicaine, comme l'a montré Jean-François Chanet dans son ouvrage L'école républicaine et les petites patries.

\section{NOTES}

1. «Une révolution comme la nôtre n'est pas un procès, mais un coup de tonnerre sur tous les méchants ", SAINT-JUST, "Rapport au nom du Comité de salut public et du Comité de sûreté générale sur la police générale, sur la justice, le commerce, la législation et les crimes des factions présenté à la Convention nationale le 26 germinal an II [15 avril 1794]», dans SAINT-JUST, CEuvres Complètes, M. ABENSOUR et A. KUPIEC (dir.), Paris, Gallimard, 2004, p. 757.

2. SAINT-JUST, L'esprit de la Révolution, 1791, dans op. cit., p. 376.

3. «On a dit que la terreur était le ressort du gouvernement despotique. Le vôtre ressemble-t-il dont au despotisme? Oui, comme le glaive qui brille dans les mains des héros de la liberté ressemble à celui dont les satellites de la tyrannie sont armés. Que le despote gouverne par la terreur ses sujets abrutis, il a raison comme despote. : domptez par la terreur les ennemis de la liberté, et vous aurez raison comme fondateurs de la république. Le gouvernement de la révolution est le despotisme de la liberté contre la tyrannie ", "Rapport sur les principes de morale politique qui doivent guider la convention nationale dans l'administration intérieure de la République ", dans CEuvres de Maximilien de Robespierre, présentation par Laponneraye, Burt Franklin, New York, t. III, 1970, p. 550.

4. Institutions républicaines, 1793-1794, dans Euvres Complètes, op.cit, p. 1139. Nous soulignons.

5. Ibid., p. 1144. Nous soulignons.

6. Les Institutions républicaines sont rédigées entre fin 1793 et juillet 1794. 
7. Nuance qui a son importance, puisqu'il n'est en aucun cas question pour Saint-Just de viser un retour à l'état de nature, les hommes modernes étant incapables, même après une réforme drastique des mœurs, de retrouver l'innocence des temps apolitiques, comme nous allons le voir. 8. Nous tenons cependant à préciser, pour éviter toute simplification, que chacune des œuvres que nous venons de citer comporte des considérations négatives et positives et qu'il n'y a pas entre elle une répartition dichotomique qui ferait des Institutions républicaines le pendant constructif du manuscrit De la nature, de l'état civil, de la cité ou les principes de l'indépendance, du gouvernement (nous l'appellerons désormais «manuscrit De la nature ») qui ne serait guère plus qu'un pamphlet de moraliste.

9. Nous entendons par là sa volonté de créer (gonos en grec : la procréation) un homme nouveau, de recréer l'homme.

10. L'esprit de la révolution, dans, op. cit., p. 399.

11. "Si tel fut l'objet du contrat social de conserver l'association, les hommes dans ce sens sont considérés comme des bêtes sauvages qu'il a fallu dompter", Institutions républicaines, dans, op. cit., p. 1093 ; " la république, par la nature de la convention, a fait un contrat politique, ou de force, entre chacun et tous et ce contrat politique forme un pacte social ; mais quelle violence, quelle faiblesse dans ce corps dénué de liaison dont le mécanisme stérile est comme un arbre dont les racines et les branches suspendues ne toucheraient pas le tronc », ibid., p. 1094.

12. De la nature, dans, op. cit., p. 1045-1046.

13. Ibid., p. 1049.

14. REFERENCES Nous soulignons.

15. Ibid., p. 1049.

16. «Discours sur la constitution de la France prononcé à la Convention nationale le 24 avril 1793 », dans, op. cit., p. 537-538.

17. Nous rappelons que pour Saint-Just, comme nous l'avons suggéré, le social et le politique constituent deux sphères antithétiques et originellement séparées l'une de l'autre.

18. L'esprit de la révolution, dans, op. cit., p. 393-394.

19. " Discours sur la constitution de la France », dans, op. cit., p. 539-540.

20. C'est en effet pour Saint-Just la dérive qu'il faut à tout prix éviter, dans la mesure où c'est justement le propre du pouvoir politique (qu'il réprouve) que de s'imposer par le biais de la figure oppressive et surplombante d'un maître.

21. Ibid., p. 540.

22. Ibid., p. 538.

23. Institutions républicaines, dans, op. cit., p. 1102.

24. On ne peut comprendre la logique et l'importance de cette thématique sans préciser qu'elle est indissociablement liée au motif des factions qui hante les réflexions de Saint-Just : devant le constat de la division croissante de la société française et de la constitution de groupes d'individus opposés à la politique du gouvernement révolutionnaire, il importe de promouvoir et même d'imposer comme une nécessité vitale la diffusion d'un sentiment fraternel d'appartenance communautaire dans la société française afin de contrecarrer en son sein tout processus de différenciation.

25. Ibid., p. 1111.

26. «Rapport au nom du Comité de salut public sur les factions de l'étranger et sur la conjuration ourdie par elles dans la République française pour détruire le gouvernement républicain par la corruption et affamer Paris ", 13 mars 1794, dans op.cit., p. 680. Les discours, parce qu'ils réagissent dans l'urgence aux évènements marquants de la révolution, sont les textes dans lesquels la radicalisation de Saint-Just se manifeste de la façon la plus nette. On remarquera que le ton se durcit tout particulièrement à partir de 1793, ce qui traduit l'impact que peut avoir l'exercice du pouvoir sur le jeune révolutionnaire.

27. Institutions républicaines, dans op. cit., p. 1103-1104. 
28. Ibid., p. 1107.

29. Ibid., p. 1100.

30. Ibidem.

31. Ibid., p. 364. Il confirme d'ailleurs cette idée dans les Institutions républicaines en déclarant que « la sagesse est dans les enfants », op. cit., p. 1089.

32. Ibid., p. 1144. Nous soulignons.

33. Ibid., p. 1139.

34. Ibid., p. 1141.

35. Ibid., p. 1135.

36. Ibid., p. 1089.

37. Georg Wilhelm Friedrich HEGEL, Philosophie de l'esprit. (1805), Paris, Puf, Epiméthée, Paris, 1982, p. 95.

38. Ibidem.

\section{RÉSUMÉS}

Pour tous les acteurs du processus, la Révolution représente en elle-même un moment de régénération collective. Mais dans l'esprit de Saint-Just, en mettant fin aux injustices et à la dépravation de l'Ancien Régime, le soulèvement de la nation n'a fait qu'ouvrir la voie à une entreprise de plus longue haleine, qui doit consister à révéler à l'homme sa « socialité » naturelle, perdue et recouverte par des siècles d'oppression et de corruption politique. Sans pouvoir rendre l'humanité à une pureté originelle définitivement perdue, les institutions républicaines doivent du moins être pensées comme les moyens privilégiés pour éduquer les citoyens de manière à les en rapprocher le plus possible.

For all the actors of this process, the Revolution epitomizes a moment of collective regeneration. But in Saint-Just's mind, putting an end to all injustices and to the depravity of the Ancien Régime through a national uprising would only pave the way for a long-term venture, the goal of which is to reveal to man his natural "sociality ", lost and covered by centuries of political oppression and corruption. Even though humanity cannot recover its original purity, republican institutions must at least be conceived as a particularly effective means to educate the citizens so as to help them get as close as possible to this lost ideal.

\section{INDEX}

Keywords : Saint-Just, sociality, institutions, Législateur, Nature

Mots-clés : Saint-Just, socialité, institutions, Législateur, Nature 


\section{AUTEUR}

\section{MARYLIN MAESO}

Doctorante à l'université Paris-Sorbonne

(Paris IV, Ecole doctorale V « Concepts et langages») 